\title{
A novel back-side light trapping structure for thin silicon solar cells
}

\section{J. Gjessing \\ jo.gjessing@ife.no}

\author{
Institute for Energy Technology, Department of Solar Energy, P.O. Box 40, 2027 Kjeller, Norway \\ University of Oslo, Department of Physics, P.O. Box 1048 Blindern, 0316 Oslo, Norway \\ University Graduate Center at Kjeller, P.O. Box 70, 2027 Kjeller, Norway
}

\section{E. S. Marstein}

University of Oslo, Department of Physics, P.O. Box 1048 Blindern, 0316 Oslo, Norway University Graduate Center at Kjeller, P.O. Box 70, 2027 Kjeller, Norway

Institute for Energy Technology, Department of Solar Energy, P.O. Box 40, 2027 Kjeller, Norway University of Oslo, Department of Physics, P.O. Box 1048 Blindern, 0316 Oslo, Norway

Decreasing silicon consumption is one of the most important ways of reducing the cost of solar cells. High-quality light trapping provides the possibility of making thinner silicon solar cells without sacrificing optical efficiency. In this work we introduce an advanced bi-periodic back-side structure with promising light trapping properties. The structure combines high coupling efficiency of light to oblique travelling modes with the ability of keeping the light within the solar cell for multiple reflections between the front and the rear side. We have done numerical simulations of light trapping for normal incidence in our structure, and we show that our structure with real materials holds the potential to exceed the light trapping of an ideal Lambertian surface. We also investigate the behaviour of our light trapping structure as a function of incidence angle and state of polarization, and compare it to the Lambertian behaviour. [D0I: 10.2971/jeos.2011.11020]

Keywords: solar cells, light trapping, diffraction grating

\section{INTRODUCTION}

Light trapping is essential in any silicon (Si) solar cell due to poor absorption in the infrared. Light trapping in industrial solar cells nowadays is commonly achieved by alkaline anisotropic etching for monocrystalline $\mathrm{Si}$ or by acidic isotropic etching for multicrystalline Si. These methods create structures with sizes that typically vary from $1 \mu \mathrm{m}$ up to $10 \mu \mathrm{m}$ depending on etch times, concentrations, temperatures etc. Because of their large and random size, these structures may be difficult to implement if the thickness of solar cells is reduced to a few tens of microns as new production methods may allow [1]. Conventional textures have been developed mainly for good anti-reflection behaviour and are not optimized for light trapping. In thinner cells, light trapping will be even more important. For thin-film solar cells light trapping is usually achieved through texturing of transparent conductive oxides (TCOs) before depositing the active solar cell material.

A benchmark for light trapping is the geometrical optics limit on path length enhancement for isotropic irradiance of $4 n^{2}$ $(\sim 50$ for $\mathrm{Si})$ in the limit of weak absorption [2]. A perfect Lambertian surface could provide such ideal light trapping. In practice, conventional light trapping structures show path length enhancements closer to 10 [3]due to lack of total randomization and absorbing surfaces. Recently it has been shown that light trapping in periodic structures can exceed the geometrical optics limit considerably at normal incidence [4]. 2D skewed pyramids [5] and more complex 3D structures [6] have already through modelling been shown to result in an increase beyond the geometric light trapping limit at normal incidence. There is experimental evidence of light trapping from periodic plasmonic structures exceeding that of the Asahi U-type glass, a state-of-the-art textured TCO glass, in thin-film solar cells [7, 8].

In this work we discuss a novel light trapping scheme consisting of a 2D periodic back-side structure as shown in Figure 1. The design has been shown through simulations to provide light trapping exceeding that of the ideal Lambertian surface at normal incidence [9]. Here we investigate the light trapping performance at nonzero angles of incidence. We also analyse the mechanisms behind the light trapping in greater detail.

\section{NUMERICAL METHODS}

The light trapping structure we have investigated and the unit cell of the grating is shown in Figure 1. The structure is based on a square lattice. The quadrants of the unit cell slopes in two different directions, so that the structure lacks the inversion of our earlier proposed structure in which the 

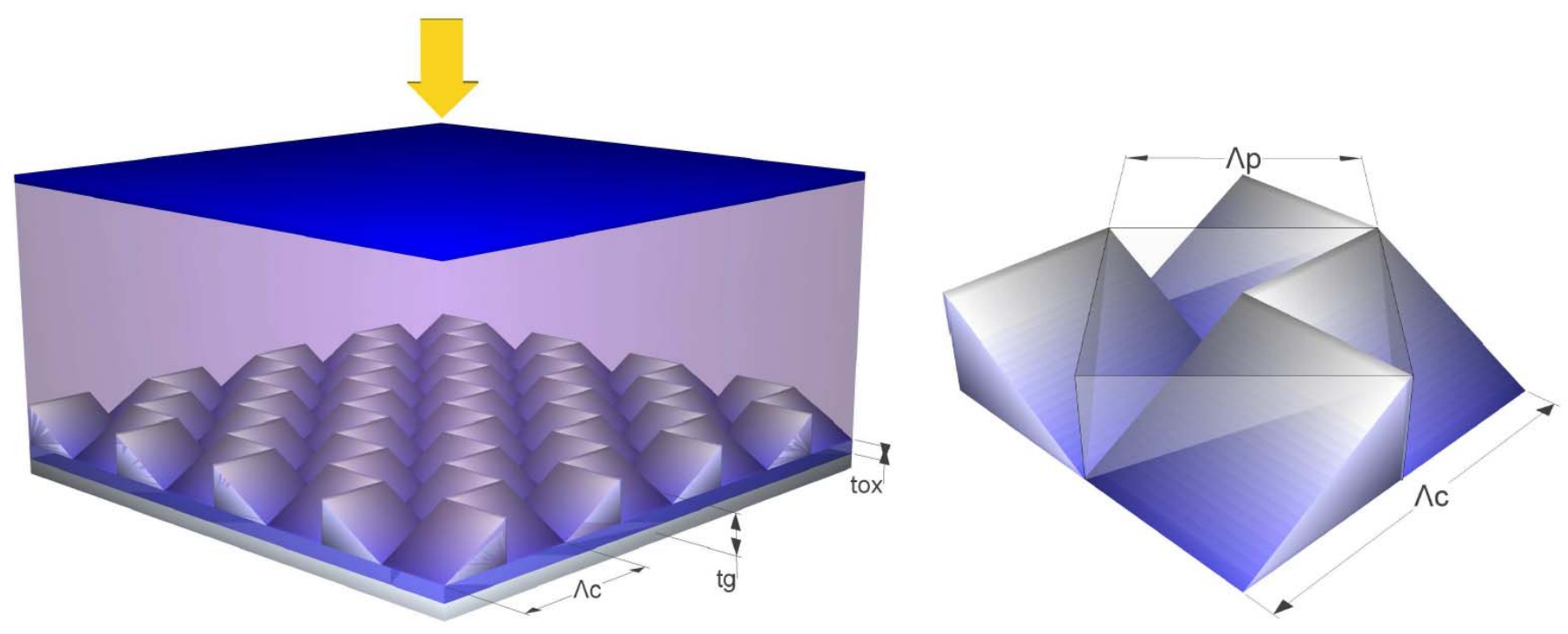

FIG. 1 Optical model for simulations (left) consisting of a planar anti-reflection coating (ARC), Si active material, light trapping layer, back-side oxide and metal mirror. The figure to the right shows the unit cell in false-colour representation. Also shown is the contour of the primitive unit cell of our structure.

oblique plane of each quadrant slopes in different directions [10]. The unit cell in false colour representation to the right also shows the contour of the primitive unit cell, which has half the area of the unit cell used in the actual calculations. The relation between the width of the primitive unit cell $\Lambda_{p}$ and the computational unit cell $\Lambda_{c}$, is given by $\Lambda_{p}=\Lambda_{c} / \sqrt{ } 2$.

Light trapping is investigated numerically by means of rigorously coupled wave analysis with the commercial software GD-Calc [11]. In GD-calc the geometrical shapes are made out of rectangular prisms. The inclined planes are thus represented numerically by the stair-case approximation using 10 steps.

A unit cell which is larger than the primitive unit cell will have a larger density of reciprocal lattice vectors than the primitive unit cell. However, only the reciprocal lattice vectors of the larger unit cell with a corresponding reciprocal lattice vector of the primitive unit cell will contribute to diffraction. Because the computational unit cell is twice the size of the primitive unit cell, we expect the diffraction efficiency of half the diffraction orders to be equal to zero. This was also what we observed in our simulations. For computations with unit cells composed of more than one primitive unit cell, our simulation software allows us to include only the non-vanishing diffraction orders in the calculations to reduce the time taken by the computations. The reduction is significant: a reduction of the unit cell area by a factor of two decreases the computation time by a factor of eight.

We have varied period $\Lambda$, grating thickness $t_{g}$ and oxide thickness $t_{o x}$ shown in Figure 1 in search of the optimal parameter combination. We calculated absorption with experimentally determined optical constants for Si [12] and for Ag and $\mathrm{Al}$ [13]. As a metric for comparing the different parameter combination we used the maximum achievable short circuit current density $J_{s c}$. A limit for the $J_{s c}$ is found by

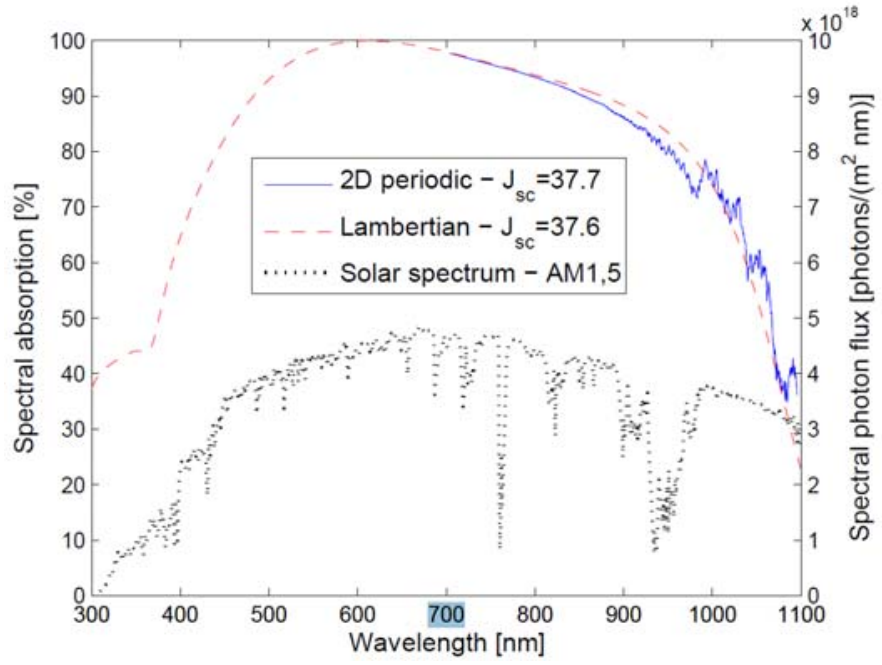

FIG. 2 Spectral absorption plot of our light trapping structure (solid, blue curve) compared to ideal Lambertian light trapping constrained by a single layer ARC (dashed, red curve). Also shown is the spectral photon irradiance (dotted, black curve).

weighting the Si absorption with the AM 1.5 global solar spectrum [14] and assuming that one electron-hole pair is generated for each photon absorbed in the Si. We integrate over the spectral distribution from 300 to $1100 \mathrm{~nm}$.

\section{RESULTS AND DISCUSSION}

A maximum $J_{s c}$ of $37.7 \mathrm{~mA} / \mathrm{cm}^{2}$ was found for the $20 \mu \mathrm{m}$ thick Si slab with Ag reflector and the parameters $\Lambda_{c}=1.4$ $\mu \mathrm{m}$ (i.e. $\left.\Lambda_{p}=\Lambda_{c} / \sqrt{ } 2\right), \mathrm{t}_{\mathrm{g}}=0.55 \mu \mathrm{m}$ and $t_{o x}=0.1 \mu \mathrm{m}$. Figure 2 shows the spectral absorption from such a structure. Al is more commonly used as reflector material than Ag in the PV industry due to lower price and more attractive electrical properties. If we substitute the Ag reflector with $\mathrm{Al}$ we find a 


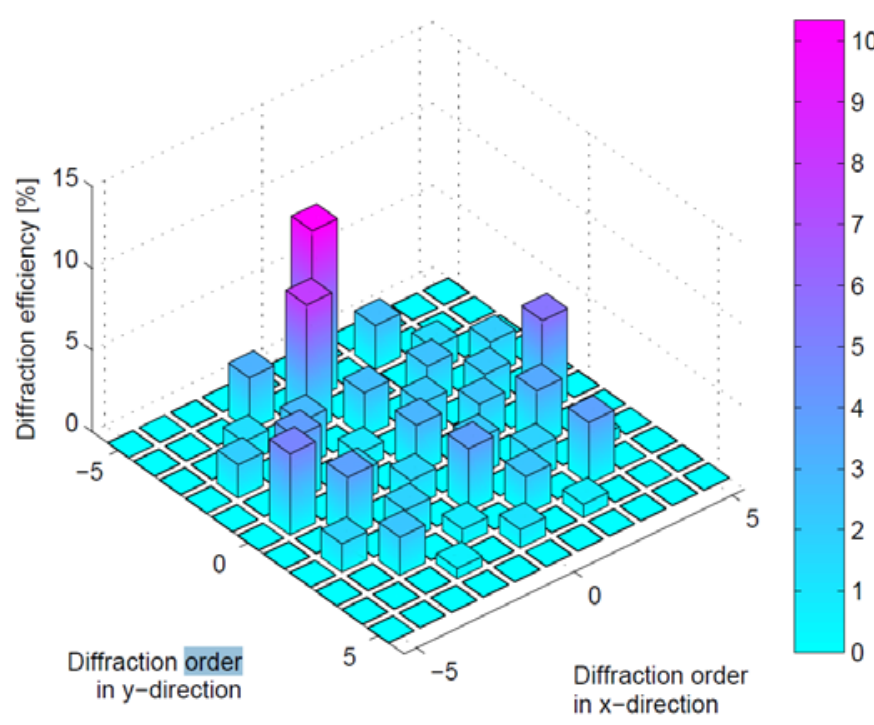

FIG. 3 Power distribution in the different diffraction orders. Every other diffraction order are zero due to the choice of unit cell.

$J_{s c}$ of $37.25 \mathrm{~mA} / \mathrm{cm}^{2}$. The decreased performance when compared to $\mathrm{Ag}$ is due to a parasitic absorption of about $1 \mathrm{~mA} / \mathrm{cm}^{2}$ in $\mathrm{Al}$ compared to only $0.4 \mathrm{~mA} / \mathrm{cm}^{2}$ in an $\mathrm{Ag}$ reflector. As a comparison it can be mentioned that the corresponding $J_{s c}$ in a planar slab without the grating structure would be $31.4 \mathrm{~mA} / \mathrm{cm}^{2}$ and $30.6 \mathrm{~mA} / \mathrm{cm}^{2}$ with an $\mathrm{Ag}$ and $\mathrm{Al}$ reflector respectively.

The low absorption observed in Figure 2 for wavelengths below $600 \mathrm{~nm}$ is due to front-side reflection from the ARC, while the absorption above $\sim 800 \mathrm{~nm}$ is limited by the combined effect of front-side reflection and incomplete light trapping. Our simulated spectral absorption curves exhibit strong and rapid oscillations resulting from interference between the front and back-side reflections in the Si slab. To make our main results evident from Figure 2, we have removed these oscillations from the curve with the help of a digital filter (a moving average).

Deckman et al. [15] derived an expression for absorption in a dielectric assuming a perfect Lambertian diffuser and no front-side reflection. To compare the light confinement in our model with Lambertian light confinement, we have made a simple adjustment to the Lambertian diffuser model. We have modified the absorption calculated with the model of [15] by adding a nonzero front-side reflection corresponding to the reflection of a $78 \mathrm{~nm} \mathrm{Si}_{3} \mathrm{~N}_{4} \mathrm{ARC}$, which is the same as we used in our structure. The resulting spectral absorption is shown in Figure 2 (red dashed line). It shows that the light trapping from our structure with Ag-reflector $\left(37.7 \mathrm{~mA} / \mathrm{cm}^{2}\right)$ exceeds our simplified Lambertian light trapping model $\left(37.6 \mathrm{~mA} / \mathrm{cm}^{2}\right)$. For wavelengths below $\sim 990 \mathrm{~nm}$, the Lambertian model exhibits more effective light trapping than ours, while our structure performs better for large wavelengths. $\lambda=990 \mathrm{~nm}$ is the wavelength where $\lambda=\Lambda_{p}$. For wavelengths with $\lambda>\Lambda_{p}$ only the zeroth diffraction order (specular reflection) can propagate to the ambient while the higher diffraction orders are totally internally reflected. This corresponds well with the observed increase in light trapping in Figure 2 at $\lambda=\Lambda_{p} \sim 990 \mathrm{~nm}$.
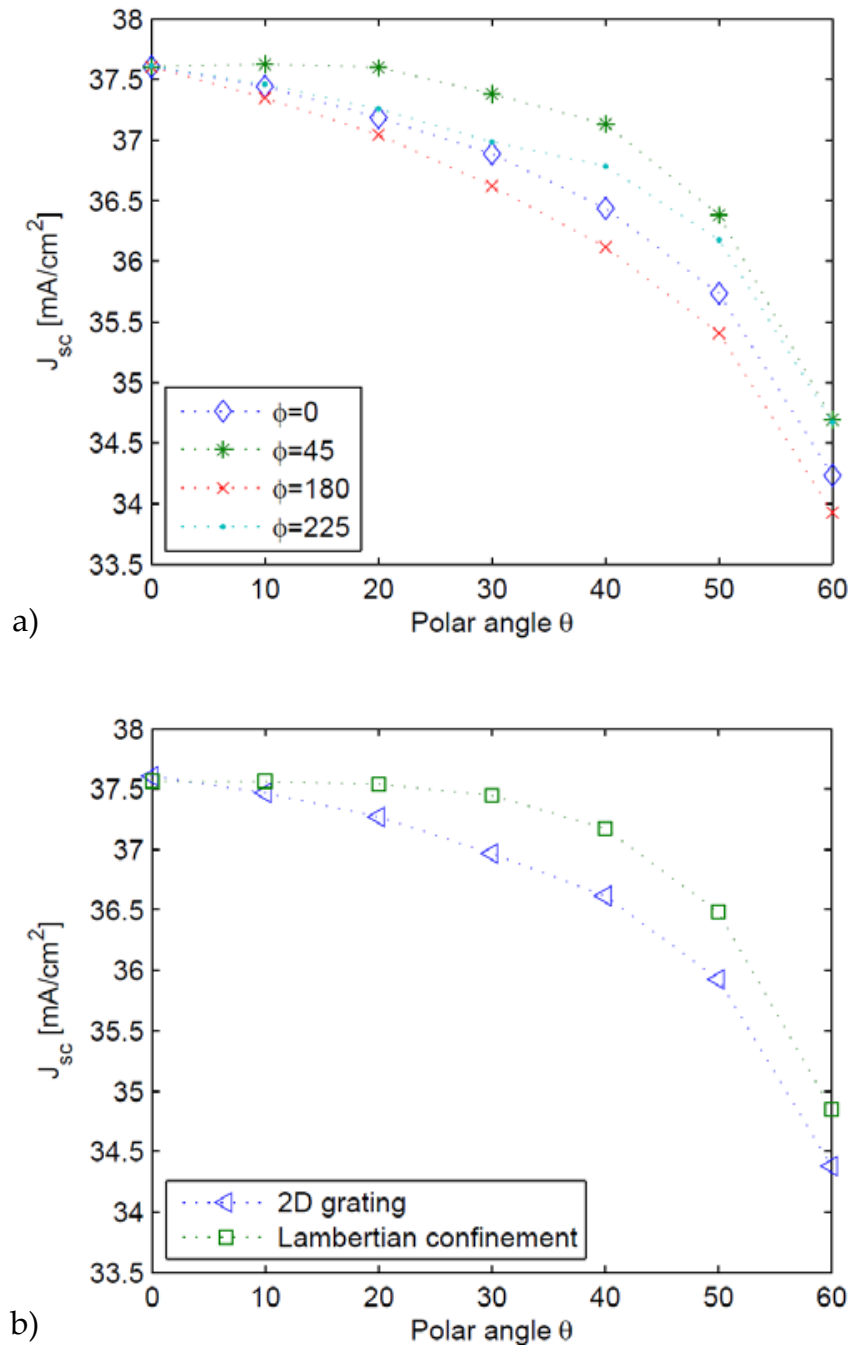

FIG. 4 Short circuit current density Jsc dependence on polar angle for four different azimuth angles a) representing the four main symmetry axes of the square lattice of the bi-periodic grating. Each point in the plot represents an average over the s-and the p-polarization. In b) and side each triangle in the graph represents an average over all four azimuth angles in a). The squares show Lambertian light trapping limited by the front-side reflectance expected from a $\mathrm{Si}_{3} \mathrm{~N}_{4} \mathrm{ARC}$ at the given incidence angle.

In Figure 3 we show the power distribution in the various diffraction orders for normally incident light at $\lambda=1 \mu \mathrm{m}$ calculated with light incident from Si. Two important properties that we have seen earlier to give good light trapping [16] are both fulfilled here: firstly, the zeroth order reflection is low (around 3\%) and secondly, light is distributed between several different diffraction orders. What is remarkable with this distribution compared to most other periodic structures is the lack of symmetry between the positive and negative diffraction orders. The reduced symmetry of this structure compared to other back-side grating structures with equivalent overall coupling to higher orders is believed to explain the success of this light trapping structure.

For higher angles of incidence the condition for total internal reflection may no longer be satisfied even if $\lambda>\Lambda_{p}$, and a reduction in $J_{s c}$ is expected. In addition, reflection from the ARC will increase for higher angles of incidence. In Figure 4a) 
we have plotted $J_{s c}$ as a function of incidence angle up to a polar angle $\theta$ of 60 degrees for four different azimuth angles $\left(0^{\circ}, 45^{\circ}, 180^{\circ}, 225^{\circ}\right)$. The different azimuth angles correspond to the four main symmetry axes of the square lattice of the biperiodic grating. Azimuth angles of $0^{\circ}$ an $180^{\circ}$ represent alignment in the direction of periodicity of the computational unit cell, while $45^{\circ}$ and $225^{\circ}$ represent alignment in the direction of periodicity of the primitive unit cell, i.e. diagonally to the computational unit cell 1 . Each curve is an average of $s$ and p-polarization. The actual solar irradiance on the solar cell is reduced by $\cos (\theta)$ which for $\theta=60$ degrees is equal to half of the irradiance at normal incidence. This geometric reduction factor is not included in Figure 4.

The advantage of Lambertian surfaces is their isotropic response. In Figure 4b) we try to differentiate the reduction in $J_{s c}$ due to higher front-side reflection from that of reduced grating performance at higher angles of incidence. The green squares show the response of the Lambertian model, limited by the front-side reflectance at the given angle of incidence. The blue triangles represents the light trapping with our structure averaged over the four curves in Figure 4a). We see that light trapping is reduced below the Lambertian limit at non-normal incidence. Most of the degradation at higher angles of incidence is due to higher front-side reflectance of s-polarized light. In addition, there are considerable variations in light trapping between the different azimuth angles. While the best curve of Figure 4a) is almost identical to the Lambertian curve, the least good are up to $1 \mathrm{~mA} / \mathrm{cm}^{2}$ lower. We can see that light trapping is better for $\phi=45$ and $\phi=225$ than for $\phi=0$ and $\phi=180$. This can be explained by the fact that $\phi=0$ and $\phi=180$ couples to more escape channels than what is the case for $\phi=45$ and $\phi=225$. Light trapping is expected to be lower when fewer escape channels exists [4].

\section{CONCLUSION}

We introduce a novel bi-periodic structure with light trapping properties matching that of Lambertian light trapping at normal incidence. A study of the coupling efficiency in the various diffraction orders reveals a non-symmetric pattern. We believe the lack of symmetry in the structure is the reason for the good light trapping achieved. We find that light trapping is reduced below that of a Lambertian structure at higher angles of incidence. Most of this reduction is caused by increased front-side reflectance for s-polarized light. The reduction in light trapping caused by less optimal grating response at higher angles of incidence is below $0.5 \mathrm{~mA} / \mathrm{cm}^{2}$ for all angles of incidence up to at least 60 degrees. A structure with light trapping property as the one we present here would render possible the production of highly efficient solar cells with thicknesses of only 20 microns. search Council and the Nordic Center of Excellence in Photovoltaic for funding this work.

\section{References}

[1] F. Henley, A. Lamm, S. Kang, Z. Liu, and L. Tian, "Direct film transfer (DFT) technology for kerf-free silicon wafering" in Proceedings of the 23rd European Photovoltaic Solar Energy Conference, 1090-1093 (Valencia, 2008).

[2] E. Yablonovitch and G. D. Cody, "Intensity enhancement in textured optical sheets for solar cells" IEEE Trans. Electron. Dev. 29, 300-305 (1982).

[3] J. Nelson, The Physics of Solar Cells (First Edition, World Scientific Publishing, London 2003).

[4] Z. Yu, A. Raman, and S. Fan, "Fundamental limit of light trapping in grating structures" Opt. Express 18, 366-380 (2010).

[5] S. E. Han and G. Chen, "Toward the Lambertian limit of light trapping in thin nanostructured Silicon solar cells" Nano Lett. 10, 4692-4696 (2010).

[6] A. Chutinan, N. P. Kherani, and S. Zukotynski, "High-efficiency photonic crystal solar cell architecture" Opt. Express 17, 88718878 (2009).

[7] V. E. Ferry, M. A. Verschuuren, H. B. T. Li, E. Verhagen, R. J. Walters, R. E. I. Schropp, H. A. Atwater, and A. Polman, "Light trapping in ultrathin plasmonic solar cells" Opt. Express 18, 237-245 (2010).

[8] H. Sai, H. Fujiwara, M. Kondo, "Back surface reflectors with periodic textures fabricated by self-ordering process for light trapping in thin-film microcrystalline silicon solar cells" Sol. Energy Mater. Sol. Cells 93, 1087-1090 (2009).

[9] J. Cjessing, A. S. Sudb $\varnothing$, and E. S. Marstein, A novel broad-band back-side reflector for thin silicon solar cells (EOS Annual Meeting, Paris, 26-28 October 2010).

[10] J. Gjessing, A. S. Sudb $\phi$, and E. S. Marstein, $2 D$ blazed grating for light trapping in thin silicon solar cells (Optics for SOLAR, Tucson, 7-9 June 2010).

\section{[11] http://software.kjinnovation.com/GD-Calc.html}

[12] C. M. Herzinger, B. Johs, W. A. McGahan, J. A. Woollam, and W. Paulson, "Ellipsometric determination of optical constants for silicon and thermally grown silicon dioxide via a multi-sample, multi-wavelength, multi-angle investigation" J. Appl. Phys. 83, 3323-3336 (1998).

[13] E. D. Palik, Handbook of optical constant of solids (Academic Press, Orlando, 1985).

[14] http://rredc.nrel.gov/solar/spectra/am1.5/

[15] H. W. Deckman, C. B. Roxlo, and E. Yablonovitch, "Maximum statistical increase of optical absorption in textured semiconductor films" Opt. Lett. 8, 491-493 (1983).

[16] J. Gjessing, E. S. Marstein, and A. S. Sudb $\varnothing$, "2D back-side diffraction grating for improved light trapping in thin silicon solar cells" Opt. Express 18, 5481-5495 (2010).

\section{ACKNOWLEDGEMENTS}

The authors would like to acknowledge the Norwegian Re- 